\title{
Anabases
}

ANABASES Traditions et réceptions de l'Antiquité

$8 \mid 2008$

Varia

\section{Aristote ou le vampire du théâtre occidental}

Entretien avec Florence Dupont

\section{Malika Bastin-Hammou et Arnaud Macé}

\section{OpenEdition}

Journals

Édition électronique

URL : http://journals.openedition.org/anabases/219

DOI : 10.4000/anabases.219

ISSN : 2256-9421

Éditeur

E.R.A.S.M.E.

\section{Édition imprimée}

Date de publication : 1 octobre 2008

Pagination : 213-224

ISSN : $1774-4296$

\section{Référence électronique}

Malika Bastin-Hammou et Arnaud Macé, «Aristote ou le vampire du théâtre occidental », Anabases [En ligne], 8 | 2008, mis en ligne le 01 juillet 2011, consulté le 20 octobre 2019. URL : http:// journals.openedition.org/anabases/219; DOI : 10.4000/anabases.219 
Anabases 8 (2008), p. 213-224.

\title{
Aristote ou le vampire du théâtre occidental ${ }^{1}$
}

\author{
MALIKA BASTIN-HAMMOU \& ARNAUD MACÉ
}

La PARUTION DE L'OUVRAGE DE FLORENCE DUPONT, Aristote ou le vampire du théâtre occidental, a suscité de nombreuses réactions, parfois enthousiastes, parfois plus critiques. Mais ces réactions ont été le fait de spécialistes d'histoire du théatre ou de la littérature, pas, à notre connaissance, d'antiquisants. Nous avons donc souhaité lui poser quelques questions relatives à Aristote et au théatre grec antique, ce quelle a aussitôt accepté. L'entretien a eu lieu à Paris, en janvier 2008.

\section{Malika Bastin-Hammou}

Nous aimerions savoir ce qui vous a amenée à écrire ce livre : vous vous êtes d'abord intéressée au théâtre latin, puis au thêâtre grec, maintenant au théâtre occidental. Quel a été le déclencheur?

\section{Florence Dupont}

Le déclencheur est vraiment ce que je dis en introduction : je me suis interrogée sur le refus de jouer la comédie romaine aujourd'hui - la tragédie à la rigueur - y compris quand le spectacle a du succès, puisque Brigitte Jacques a eu un succès fou avec l'Aululaire, ensuite le Pseudolus. Or ces succès n'ont pas engagé les directeurs de thêâtre à accueillir de nouvelles pièces de Plaute. Il y a une résistance, que j'essaie de comprendre. Donc j’ai mis en relation le travail de certains chercheurs sur la comédie romaine - notamment celui de Pierre Letessier en France, et la façon qu'ont les metteurs en scène d'aborder les textes. Il y avait deux points qui achoppaient : le fait que la comédie romaine, le thêâtre latin, soit un thêâtre rituel, ce que personne n'avait jamais pris en compte précédemment ; et le fait que tous les textes de thêâtre sont lus de façon narrative. Il fallait donc aller chercher aussi loin qu'il était nécessaire pour comprendre les raisons de cette situation.

1 Entretien avec Florence Dupont, à propos de son dernier ouvrage Aristote ou le vampire du théâtre occidental, Paris, Aubier, Collection «Libelles ", Paris, 2007. 
Il m’a semblé, en écoutant les étudiants de théâtre, qu'il y avait une vulgate aristotélicienne qui prévalait, et que si tout le monde - étudiants, enseignants, professionnels du théâtre, surtout professionnels du théâtre - se référait tout le temps à la Poétique d'Aristote, ce n'était pas par souci d'érudition, mais parce que la Poétique d'Aristote constitue l'origine mythique du thêâtre occidental. En fait, ce n'est pas au texte lui-même que la plupart se réfere mais à une vulgate aristotélicienne, un aristotélisme contemporain. C'est pour ça que j’ai voulu publier mon livre dans la collection « Libelles ", en espérant que son audience dépasserait celle des spécialistes de l'antiquité grecque.

MBH : Et Avignon 2005, était-ce aussi un déclencheur?

$F D$ : Non, pas vraiment, parce que j'y travaillais depuis longtemps; et c'est en travaillant que je suivais les événements, et je me disais "Ce n'est pas possible ! j'ai raison d'écrire ce livre ! ». Puis $j$ 'ai pris beaucoup de retard dans mon travail, je voulais tout vérifier. Il aurait dû paraitre plus tôt, donc, Avignon 2005 n'a fait que confirmer ce que j'étais en train d'écrire.

$M B H$ : Vous soutenez que l'aristotélisme fait des ravages au thêatre : est-ce que cela vaut, selon vous, pour tous les thêâtres ou seulement pour le thêâtre public - type Avignon ?

$F D$ : Je crains que ce ne soit une conception majoritaire du thêâtre. Tout le monde semble convaincu que le théâtre est la représentation d'une histoire. Cette conception étroite est propre à l'Occident et s'accompagne d'une série d'idées toutes faites : comme celle selon laquelle la Grèce est à l'origine du théâtre - tout le monde ignore le théâtre indien, japonais, chinois. Politiquement, c'est insupportable. Un de mes étudiants japonais, qui travaille sur le thêâtre baroque français, s'étonnait de notre ethnocentrisme. C'est comme ça que j’ai été amenée, à sa demande, à écrire des articles dans des revues japonaises sur le thêâtre romain. Cet aristotélisme contemporain est une forme de néo-colonialisme. C'est ainsi que des étudiants, venus du Maghreb ou d'Afrique, où il n'y a pas de tradition théâtrale, travaillent sur des spectacles rituels afin d'imaginer un thêâtre contemporain qui se distingue du thêâtre d'Aimé Césaire, encore esthétiquement prisonnier de l'aristotélisme.

Pour moi, l'enjeu politique est important. Rompons avec ce mythe d'un théâtre grec origine et modèle du thêâtre universel... En plus, ce mythe fait partie d'un bloc : à l'invention dionysiaque du théâtre en Grèce est associée l'invention de la démocratie, le tout empaqueté avec Aristote, alors qu'Aristote n'a rien à voir avec l'un ou l'autre. Vous me dites le thêâtre privé ?

$M B H$ : Le thêâtre privé me semble moins obsédé par la quête du sens, du message, et moins soucieux d'aristotélisme.

$F D$ : Ce n'est pas parce qu'ils ont rompu avec l'aristotélisme que les metteurs en scène du thêâtre privé n'y font pas référence. En revanche, l'idéologie aristotélicienne concerne moins des expériences très marginales, les expériences comme celles des chorégraphes qui travaillent avec des metteurs en scène. Malheureusement, il ne s'agit pas de spectacles populaires. En revanche, le vaudeville, lui, n'est pas aristotélicien. Plus généralement, c'est le thêâtre méprisé qui échappe à l'aristotélisme. 
$M B H$ : Donc le théâtre privé ? N'est-ce pas une spécificité française, due à l'institution du thêâtre public qui est très liée à l'éducation nationale ?

FD: Les Allemands disent la même chose de leur propre théâtre. J'ai eu la chance de suivre une expérience très intéressante à Genève au théâtre du Grütlli. Une metteure en scène, originaire de Vienne montait les Perses, c'était très intéressant, très beau, mais finalement une variante de cette idéologie aristotélicienne... Tout était tiré du texte. Elle n'avait pas envisagé l'hypothèse qu'il pouvait y avoir une structure autre que narrative... Le chœur n'existait pas ou plutôt, elle avait demandé à des citoyens de Genève de faire le chœur depuis la salle. Ce chœur, bien sûr, ne chantait pas. Je ne suis pas adversaire de ce genre d'expériences, je regrette simplement qu'elles s'enferment dans l'aristotélisme.

$M B H$ : Ne pourrait-on expliquer les raisons de ce primat accordé au texte en allant chercher du côté de la formation des metteurs en scène?

$F D$ : Les grandes ruptures, pour moi, ont eu lieu au XVIII ${ }^{\mathrm{e}}$ s., avec la déritualisation du thêâtre, puis au siècle suivant avec l'invention de la mise en scène, qui mettent le texte et la lecture du texte au centre de tout. C'est pourquoi j'ai proposé ce parcours à l'intérieur de l'histoire européenne du thêâtre, $\mathrm{XVIII}$-XIX ${ }^{\mathrm{e}} s$.

On voit progressivement, avec les Lumières, le théâtre se déritualiser ; l'acteur de théâtre n'est plus quelqu'un de différent, mais devient un citoyen comme un autre et le théâtre se défait peu à peu, tel qu'il était constitué traditionnellement, et rien ne remplace sa fonction sociale. Ce que je pense, c'est que dans la culture républicaine et démocratique le théâtre n’a pas de place prédéfinie, ce n'est pas une institution, il doit toujours se justifier : car on peut faire désormais ce qu'on veut au théâtre.

$A M$ : Est-ce pourtant aujourd'hui au théâtre que se manifeste le plus le souci aristotélicien de la bonne facture des histoires? Vous qui avez travaillé sur les séries, avec Homère et Dallas, ne trouvez-vous pas que le cinéma, et plus encore l'écriture de la série, est un lieu où l'auteur peut concevoir son travail comme celui d'un artisan œuvrant à la production d'une histoire?

$F D$ : Vous connaissez, vous savez comment travaillent les scénaristes?

$A M$ : Oui.

$F D$ : Je n'ai pas la même expérience, pour la télévision. Mais à ce que je sais, c'est que la question, ce n'est pas la bonne histoire. Aristote dit, par exemple, que le poète ne doit pas se soumettre à la dictature de la clepsydre qui définit le temps imparti à la performance. Le temps d'une tragédie doit être déterminé par l'intrigue. Dans une série, c'est l'inverse. Chaque épisode doit tenir dans une durée bien définie. Ensuite, les personnages ne sont pas déduits de l'histoire. J'aurais tendance à penser que les Français font de mauvaises séries justement parce qu'ils privilégient l'histoire, ils créent un lien narratif d'un épisode à l'autre. Dans la série américaine, il n'y a pas de continuité d'un épisode à l'autre, on peut les projeter dans le désordre... 
$A M$ : C'est dans la sitcom qu'il n'y a pas de continuité narrative d'un épisode à l'autre, mais la série quant à elle déploie au contraire son récit sur un temps long, celui d'une saison, voire de plusieurs saisons. Ainsi, par exemple, avec les Sopranos, qui sont peut-être le modèle de la façon dont la série contemporaine a apprivoisé le temps long comme espace dramatique.

FD: On ne peut pas dire des Sopranos «c'est une bonne histoire». C'est d'abord des personnages. Si vous changez les personnages à cause de l'histoire, vous ne vous en sortez pas et c'est le propre des séries françaises. Car le récit est secondaire. Il fait fonctionner les personnages, mais pas l'inverse. Vous savez très bien que la seule fonction d'un feuilleton, c'est d'amener la pub avant et après.

$A M$ : Il y a néanmoins une évolution, au milieu des années 90, entre Dallas, Dynastie, les Soap operas et les séries comme les Sopranos, Six feet under, tout ce qui est venu avec HBO, et qui a donné au récit une nouvelle dimension.

$F D$ : Vous connaissez les séries allemandes?

$A M:$ À part Derrick, je dois avouer...

$F D$ : C'est très intéressant parce que c'est un bon exemple de série. Même si une forme de la narrativité s'introduit, qui établit un fil conducteur - vous avez raison - je ne pense pas que la série puisse être définie comme la représentation d'une histoire commençant avec le premier épisode et se terminant avec le dernier.

$A M$ : Que pensez-vous de l'hypothèse de Jacques Rancière, qui n'est pas contradictoire avec la vôtre, mais selon laquelle Aristote revient dans la modernité par Hollywood? Rancière distingue dans l'histoire de l'art trois régimes qui sont autant de façon pour l'art de signifier : régime pré-aristotélicien, éthique et poétique ; régime aristotélicien par lequel l'art s'accomplit dans la représentation des histoires ; régime esthétique introduit par l'époque romantique, qui cherche un surplus de sens au-delà de la simple narration. Or, Hollywood, le système des studios, c'est le retour du représentatif, d'Aristote, au cœur de l'âge esthétique.

$F D$ : Le problème, c'est qu'Aristote ne s'intéresse jamais au sens, seulement aux passions suscitées par le muthos.

$A M$ : Exactement : Aristote n'exige pas d'autres sens que celui de l'histoire et des affects qu'elle suscite. Or c'est cela qui revient avec Hollywood: l'obsession de bien raconter des histoires et de les voir faire de l'effet. Dans l'Hollywood classique, disons, par exemple, avec John Ford, la mise en scène doit être efficace, c'est un travail d'artisan, sans auteurisme. Voici ce qui fait l'aristotélisme d'Hollywood - comment bien écrire des histoires, comment les mettre en scène en bon artisan - alors que les scènes théâtrales me semblent de ce point de vue bien loin d'Aristote, marquées par un véritable auteurisme de la mise en scène.

FD: Mais qu'entendez-vous par le récit au cinéma? Le scénario? Alors on est loin d'Aristote. 
$A M$ : Pas seulement le scénario. La mise en scène des histoires.

$F D:$ Si on reprend Aristote, le muthos est une forme abstraite, et ensuite il y a le texte. Dans le parallélisme avec le cinéma, c'est le film qui devient « le texte ». Alors il n’y a plus de mise en scène. Au cinéma, il n'y a pas de mise en scène, de spectacle, d'opsis. Si le spectacle consiste à faire voir un texte, il y a quelque chose en plus que le texte. Quel est l'équivalent du texte au cinéma ? Soit le scénario, soit le film. Entre les deux, je ne vois pas. Ce précédent à la performance, fondamental pour Aristote, qu'est le texte, est absent du cinéma. La comparaison ne marche pas.

$A M$ : Peut-être y a-t-il cette différence, mais quand Ford se pose des questions de mise en scène, il se pose des questions aristotéliciennes, d'agencement des actions et peinture des caractères : il se demande comment son cow-boy doit entrer dans son saloon, en sortir, afin que l'histoire qu'il doit raconter soit efficacement racontée, que l'on en comprenne la logique et que les caractères soient campés avec clarté.

FD: C'est de l'écriture par l'image. Il est comme le poète qui écrit le texte.

$A M:$ En effet : il s'applique à bien raconter une histoire, il est aristotélicien.

$F D$ : C'est le passage du scénario au texte-performance qu'est un film : le scénario est l'bupokrisis, non pas le muthos qui est une forme abstraite. Le poète selon Aristote est un fabricant de " muthoi» et non pas un artisan de mots. En fait, au cinéma, il n'y a rien d'autre que la pellicule, c'est la même chose que le texte. Lire un livre ou faire passer une bobine, c'est pareil. En fait, il n'y a pas d'interactivité. Là où le cinéma est semblable à Aristote, c'est que le cinéma sépare le public de l'événement. Jusqu'à la fin du XVII siècle, jusqu'à la salle à l'italienne, le teatro ne sépare pas la scène du public : quand on parle de theatrum mundi, ce n'est pas ce qui est représenté sur scène mais ce qui se passe, l'événement thêâtre. Mais Aristote n'en parle pas. Je suis d'accord avec vous pour dire qu'Aristote est moderne, contemporain, proche du cinéma. Mais il l'est en détruisant le thêâtre du jeu.

$M B H$ : Pourrait-on revenir sur ce que vous identifiez comme une rupture dans l'histoire du thêâtre, c'est-à-dire l'émergence de la figure de l'écrivain de théâtre, que vous attribuez à Goldoni ? Je trouve votre analyse très intéressante, en particulier la mise en contexte sociologique et économique. Mais n'est-ce pas récurrent, historiquement, cette volonté qu’ont les auteurs de thêâtre de se distinguer du reste de la troupe, en particulier des acteurs ? Je vois chez Goldoni ce que je vois déjà chez Aristophane : une volonté de déposséder les acteurs de leur statut pour construire celui de l'auteur.

$F D$ : Oui, bien sûr. Mais ce que vise l'auteur est différent. Ce à quoi vise Goldoni, c'est à une reconnaissance sociale parmi les « auteurs » littéraires. En Grèce, le poète tragique comme le poète comique veulent être vainqueurs dans un concours et non pas avoir leurs livres dans une bibliothèque.

$M B H$ : Le poète comique veut cesser d'être un kômôdos pour devenir un poiètès. 
$F D$ : Oui, mais le problème, c'est que la catégorie du poiètès, que signifie-t-elle du point de vue de la dignité sociale? Elle n'est pas exportable en dehors de la Grèce. Goldoni ne veut plus être au service d'une troupe où il est le plumitif qui écrit des canevas, il veut son nom parmi les livres des bibliothèques. Il me semble que pour Aristophane, la gloire sociale ne sera pas du même ordre. Autre exemple, à Rome cette reconnaissance publique du poète dramatique est tout à fait absente. D'ailleurs il n'y a jamais eu de gloire à Rome pour les auteurs de théâtre. Au contraire, certains prennent plaisir à humilier les rares nobles qui prétendent écrire. Certes, les textes des auteurs de thêâtre sont bien récupérés dans les litterae latinae, mais ils serviront aux exercices pour les orateurs ou ils fourniront des citations dans leurs discours, mais les poètes n'en tirent aucune gloire.

$M B H$ : Il faut, en Grèce, distinguer comédie et tragédie. Il y a bien, au Ve siècle, une reconnaissance sociale pour les poètes tragiques - d'ailleurs, des personnages de haut rang, comme Sophocle, la pratiquent avec gloire. Cette reconnaissance sociale est en revanche refusée aux poètes comiques, ce contre quoi s'élève un Aristophane, et cela s'inscrit dans ses textes : jamais il ne s'y désigne comme kômôdos - terme qui désigne celui qui fait des comédies, qu'il s'agisse d'un acteur, d'un choreute ou d'un auteur ; il lui préfere le terme de poiètès, qu'il n'emploie par ailleurs que pour les poètes tragiques.

$F D$ : Les Grandes Dionysies sont une institution politique où il convient qu'il y ait un vainqueur. Il n'y a pas l'équivalent à Rome. Et pas de reconnaissance sociale associée au thêâtre sauf pour l'organisateur des jeux. La relation patronus-poète fondée sur le don et le contre-don (le poète donne des poèmes au patron qui lui donne une maison, une propriété, de l'argent), personne n'offre une tragédie ou une comédie. Le théâtre est à part. Le " poète " dramatique n’est pas reconnu socialement comme poeta, même si le mot est présent chez Térence et Plaute. Le poeta est un personnage. C'est un des actants du spectacle. Le terme poeta, terme grec utilisé à Rome, n'est pas valorisant. À l'époque augustéenne, le terme de vates lui sera substitué, quand la pratique poétique deviendra une fonction sociale reconnue. Le poeta est un scribouillard qui en plus ne gagne presque rien.

$M B H:$ Du coup, puisque la configuration est différente, on peut imaginer que les buts visés par les poètes ne sont pas les mêmes. À Rome, s'il n'est pas question de gloire, seul le rituel compte. En Grèce, d'accord il faut faire du rituel, mais il y a autre chose : la quête d'une reconnaissance sociale, par exemple, et sans doute économique.

$F D$ : À Rome la gloire revient à celui qui préside les jeux. Soit dans sa fonction de magistrat, soit comme privé.

$M B H$ : D'accord. Mais alors, pourquoi le poète fait-il du théâtre?

$F D$ : Pour gagner de l'argent. C'est un intellectuel précaire.

$M B H$ : Mais alors, qu'est-ce qui permet d'en gagner plus que l'autre?

$F D$ : C'est selon que l'éditeur des jeux a plus ou moins d'argent. 
$M B H:$ Et cet élément ne s'inscrit-il pas dans le texte?

$F D$ : Non. C'est un scénariste. Il fournit ce qu'on lui demande. Prenons l'exemple des jeux privés. On demande à un chef de troupe, qui a généralement ses poètes, de produire une pièce.

En plus, les poètes ne sont payés quaprès le spectacle. Si le rituel a échoué, ils ne sont pas payés. Tout se passe entre le chef de troupe et le noble qui donne les jeux.

$M B H$ : Sur cette question du rituel qui échoue... vous citez des passages de comédie, "maintenant vous pouvez vous lever, vous pouvez applaudir " : ne peut-on imaginer que ces vers sont ludiques? Le poète ne joue-t-il pas, ici, avec le rituel, et la possible impatience des spectateurs?

$F D$ : Pas du tout ! Il y a des exemples historiques où le spectacle a vraiment été interrompu. C'est la fameuse histoire de Clodius qui a interrompu les spectacles qui se donnaient lors des jeux de la Grande Mère. Avec pour conséquences des prodiges effrayants sur la rive droite du Tibre. L'haruspice consulté sur ces prodiges répond que "c'est à cause de l'interruption des jeux ". Certes, le poète en joue, comme d'une possibilité, un risque, mais un vrai : car si les gens se lèvent, ils deviennent ceux qui arrêtent les jeux.

$M B H$ : Je trouvais ça un peu contradictoire avec l'idée d'un public très vivant : je pense à ce qu'Aristophane imagine dans les Oiseaux - que les chœurs tragiques sont parfois bien ennuyeux, et que s'ils avaient des ailes, les spectateurs s'en iraient faire un tour chez eux en attendant qu’ils soient terminés.

$F D$ : Ce n'est pas contradictoire. La ritualité n'exclut pas la vie, il suffit de regarder les rituels dans les sociétés traditionnelles. Car ce n'est pas la réalité qui compte, mais c'est toujours la parole du prêtre qui dit ce qui est. C'est la parole de celui qui préside les jeux, il est en costume de Jupiter, c'est Jupiter qui préside les jeux. C'est l'histoire du vieillard qui chante. Toute interruption des jeux est une grave faute religieuse. Un jour on annonce l'arrivée des Carthaginois près de Rome. Tout le monde se lève, pour prendre les armes, et les jeux seraient interrompus avec tous les risques religieux que cette interruption causerait... Mais un vieillard a continué à danser dans le théâtre, avec un joueur de flûte. Quand les Romains sont revenus au théâtre pour voir ce qu'il en était, ils ont eu une formule religieusement efficace devenue proverbiale, Salva res est, dum saltat senex, «Tout va bien, le vieillard danse ».

\section{$A M: \mathrm{Y}$ a-t-il la même dimension dans les Grandes Dionysies?}

$F D$ : Je ne suis pas spécialiste de religion grecque; ce qui m’intéresse, c'est qu'il y a un cadre rituel, ou social, qui crée des contraintes au spectacle, qui ne passent pas par la fonction narrative, et auxquelles doit au contraire s'assujettir la fonction narrative. On ne peut pas oublier le public, les circonstances, la société grecque. D'où une certaine codification avec laquelle le poète ou les acteurs peuvent jouer, mais qui ne doit pas être transgressée, jouer n’est pas transgresser. 
$M B H$ : Cette réévaluation de la musique et du public fonctionne très bien quand c'est appliqué à Molière. Est-ce vrai seulement du Bourgeois, ou cela fonctionne-t-il pour d'autres pièces?

$F D$ : C'est vrai de toutes les comédies ballet. L'abandon de la musique est vraiment récente. Les auteurs classiques se sont construit leur Aristote, qui n'est pas notre Aristote " ultralibéral ", déritualisé. Ils avaient un théâtre extrêmement codifié. Cet aspect-là, ils ne l'ont pas repris à Aristote.

Bien sûr, toutes les pièces de Molière ne sont pas musicales. Mais Pierre Letessier a montré que la fonction narrative ou représentative, dans Tartuffe, n'était pas première : il ne s'agit pas de raconter une bonne histoire, ni même de représenter des mœurs, mais de construire du spectacle à partir de formes convenues et mises en variation... C'est plus difficile à faire admettre. Car il $\mathrm{y}$ a tous ces discours qui cherchent à donner une légitimité morale au théâtre... C'est comme aujourd'hui, il y a d'excellents metteurs en scène qui tiennent des discours aberrants. Sobel nous abreuve de propos politiques, on s'attendrait à un thêâtre pédagogique et militant, puis il fait de superbes spectacles.

$M B H$ : C'est peut-être ce qu'on attend de lui, institutionnellement ?

FD: Pour Sobel, c'est plus profond. Il n'est pas machiavélique, non. C'est toute la tradition brechtienne.

$M B H$ : Et pour ce thêâtre classique, peut-on encore parler de fonction rituelle?

$F D$ : En tout cas de fonction sociale. Les théâtres existent par privilèges du roi. Ce qui donne un statut social, culturel, au théâtre, même si cette idée révulse les belles âmes. C'est ce qui m’a intéressée. Le théâtre avant la nuit du quatre août. Puis le théâtre est devenu une marchandise. J'avais prévu de parler dans ce livre du théâtre indien, mais c'était beaucoup trop long. Du coup on est pris, c'est une erreur de ce livre, dans le duo infernal Grèce-Rome. Mais, les thêâtres rituels, il y en a de toute sorte. Avec des systèmes complètement différents.

$M B H$ : Peut-on revenir sur les critiques que formule Denis Guénoun dans l'article du Monde des livres qu'il consacre à votre ouvrage ? En particulier cette idée que le primat du texte au théâtre, dont l'origine remonterait à Aristote, permettrait de sortir de la fonction identitaire de la tragédie au profit de la quête du sens, sortie qui serait peut-être salutaire d'un point de vue, du moins, politique?

$F D$ : Il y a une volonté politique, chez Guénoun, une volonté universaliste. Il s'agit d'abolir les différences grâce à la littérature - la littérature française. Le thêâtre qưil aime est un lieu du sens. Mais lorsqu'il veut montrer que c'est vrai du théâtre en général, je ne le suis pas. Guénoun veut réconcilier tout le monde, en prétendant qu'il n'y a que du théâtre du sens, que du théâtre du texte - le premier étant le théâtre tragique des Grecs. C'est chez lui une option politique dont il pense qu'elle est émancipatrice. Pour moi elle est impérialiste. 
La fonction identitaire de ce thêâtre, oui, on peut la déplorer, mais affirmer que cela n’a pas eu lieu historiquement, c'est autre chose. Le problème, c'est toujours ce statut de la Grèce ; si les Grecs, c'est nous, alors on a un problème. Mais il n'y a aucune raison que les Grecs soient nous.

$M B H$ : Et pourtant vous en appelez au retour d'un thêâtre identitaire et rituel ?

$F D$ : Pourquoi est-ce que j’aime tant les conventions, le théâtre codifié ? C'est que cela permet une autre présence du public, le public comprend ce qui se passe, participe au jeu avec le code, c'est plus vivant. Il y a, par exemple, une question actuelle : comment jouer le Vaudeville ? Jouer en rupture avec la codification propre au Vaudeville, c'est dire au public : "Vous êtes des abrutis qui attendez un théâtre de la digestion » En revanche, l'auteur ou le metteur en scène peut proposer au public de jouer ensemble avec les codes du Vaudeville - il ne s'agit pas de refaire indéfiniment $A u$ théâtre ce soir. C'est ce qu'a fait Koltès dans la première mise en scène du Retour au Désert, en donnant à Jacqueline Maillan le rôle de Mathilde, en jouant ainsi, hors du Vaudeville, avec les codes du Vaudeville. Cette pièce jouée autrement devient ennuyeuse et incompréhensible. Voilà ce que j'appelle un théâtre codifié, plutôt que rituel, avec un code partagé, et un jeu possible sur ce code, avec le public. Ça peut passer par la réutilisation au thêâtre d'autres codes spectaculaires, comme ceux que les gens voient à la télévision, au cinéma.

$M B H$ : L'opposition que vous utilisez entre élitiste et populaire ne recoupe-t-elle pas celle que vous faites entre dramatique et codifié?

FD: Cela ne se recoupe pas nécessairement au cours de l'histoire du théâtre, mais cela tend à le faire aujourd'hui, avec la prépondérance de cette idée que le sens du spectacle doit passer par l'histoire, par le drame, par l'action. Et en même temps, actuellement, il existe un théâtre nondramatique, théâtre d'avant-garde comme on disait jadis, ou encore post-dramatique qui n'est pas du théâtre codifié, mais qui est élitiste. On le joue en général, paradoxalement, dans les banlieues, ce sont des pièces sur les licenciements, la mondialisation, faisant usage d'une métathéâtralité exhibée. On peut penser à Heiner Müller. C'est un thêâtre intellectuellement très intéressant, mais sans plaisir spectaculaire, on est toujours esthétiquement dans la rupture, la transgression, la déconstruction. Ce genre de thêâtre dit au public : "Vous attendez du plaisir, eh bien on va vous le refuser $\%$. Si aujourd'hui un spectacle n'a pas de structure dramatique, aucune autre structure ne donne son cadre au spectacle, un cadre qui par exemple justifie la fin de la pièce - j'ai vu récemment ainsi Du boulevard, du boulevard, de Mesguich, à la fin il amenait un canon sur scène, pour bombarder sa pièce, il n'avait pas d'autre solution pour la terminer.

$A M$ : Mais n'y a-t-il pas une dimension narrative forte dans ces genres populaires? Cette dimension même qui a été étudiée par Aristote, lorsqu'il se demandait dans la Poétique comment il fallait raconter une bonne histoire?

$F D$ : Il y a bien sûr des histoires, par exemple dans le Vaudeville, mais le genre est premier, c'est-à-dire le code : on vient voir tel type d'acteur, tel type de situations connues à l'avance, et c'est tel type d'acteur qui fait bien fonctionner l'histoire, non l'inverse. On n'est donc pas dans un système aristotélicien. Notez bien que je n'ai jamais dit qu'il ne fallait pas d'histoires, de bonnes histoires. Simplement, pour moi, dans un théâtre codifié, les histoires et le texte ne sont 
faits que pour que ce type de thêâtre fonctionne, conformément aux attentes du public, avec les acteurs et le code adéquats. Le texte n'est fait que pour être joué par tels types de comédiens, par exemple, ceux du Vaudeville, dont l'auteur sait à l'avance qu'ils vont « hyperjouer ». Dire cela s'oppose à la vision d'Aristote pour lequel tout le plaisir de la pièce peut être pris à la lecture de celle-ci.

$A M$ : Une des raisons que vous donnez à cette attitude d'Aristote face à la tragédie est le fait qu'il soit étranger et qu'il ne se reconnaisse pas dans ce que vous voyez comme un rite athénien. Mais le fait qu'Aristote choisisse d'insister sur le muthos, sur le récit à l'œuvre dans la pièce et dont l'agencement peut être saisi dès la lecture, au détriment des autres éléments du spectacle, ne pourrait-il pas aussi trouver sa source dans l'évolution même du genre tragique? Aristote présente ainsi l'évolution de la tragédie au $V^{e}$ siècle, en décrivant l'introduction progressive d'un plus grand nombre d'acteurs comme une tendance propre à permettre l'épanouissement de la dimension narrative.

$F D$ : Cette idée d'Aristote ne correspond à aucune réalité historique. La place de la musique aussi s'accroît, par exemple. Et cette idée même d'une évolution est arbitraire. Il y a des variantes multiples, mais pas d'évolution linéaire. Même la multiplication des dialogues, qui correspond en effet peut-être à un goût du public, ne signifie pas nécessairement que le muthos devient plus important.

$A M$ : Ne faut-il pas en outre tenir compte du fait qu'Aristote a une méthode qui lui impose de tenir un discours propre à chaque genre de choses, avant de s'étonner de ne pas trouver de référence au contexte social dans la Poétique? Au livre VIII des Politiques, l'effet social du spectacle l'intéresse. Ici, dans le genre poiètique, il ne s'intéresse qu'à la fabrication de l'histoire par l'auteur et ne s'adresse qu’à ceux que cette fabrication intéresse.

$F D$ : Le fait qu'Aristote s'adresse à un tel public est une fiction. Ce traité ne s'adresse à personne. Dans l'antiquité, aucun traité technique n'est un vrai traité technique. Pas plus l'Art d'aimer que les Géorgiques, ou les manuels de rhétorique, n’ont de but pédagogique. Il s'agit plutôt de donner un statut théorique ou philosophique à un certain type de pratiques, quitte à les déformer.

$A M$ : Néanmoins, indépendamment du but qu'ils fixent à leurs traités, Platon, dans le Phèdre, par exemple, et Aristote dans la Poétique, cherchent à décrire un état de la pratique des auteurs tragiques. Ne pensez-vous pas qu'Aristote pourrait simplement témoigner du fait que dans la pratique même les auteurs se soient préoccupés davantage des histoires que de la construction des décors ou d'autres éléments du spectacle qui relevaient, comme le dit Aristote, de la compétence d'autres métiers?

$F D$ : Qu'il y ait eu une autonomisation des pratiques d'écritures est un fait, mais vous faites comme si le but d'Aristote pouvait être de décrire une situation, ce qui n'est absolument pas le cas. Car sa Poétique est une pure fiction.

$A M$ : Ce sont tout de même des notes de cours que nous avons là. 
$F D$ : La question est de savoir pourquoi on publie des notes de cours, est-ce que vous publiez vos notes de cours, vous?

$A M$ : Mais il ne les a pas publiées, justement.

$F D$ : Oui, mais il y a des gens qui l'ont fait, et la question est de savoir pourquoi. Et pourquoi il fait ce genre de cours, car il ne fait pas cours à des futurs poètes tragiques! Qu'il y ait depuis le Ve siècle une autonomisation de la pratique de l'écriture chez les auteurs tragiques, c'est une chose, seulement Aristote n'est pas là pour en rendre compte, mais pour l'utiliser. Et il fait la même chose avec la rhétorique. Le traité sur la rhétorique d'Aristote est tout sauf quelque chose qui puisse servir à qui que ce soit.

$A M$ : Si le texte d'Aristote est pour vous avant tout quelque chose qui fait un usage délibéré d'éléments contemporains en visant ses propres buts, il faut néanmoins reconnaittre qu'une prétention à décrire objectivement les choses et les pratiques de son temps fait partie de cet usage.

$F D$ : Mais il ne décrit aucune réalité objective et ce dont il parle n'est pas la pratique du thêâtre de son temps ni du V viècle. Comme vous le dites, il dit comment il faut faire. C'est un traité de déontologie, mais dans l'abstraction.

$A M$ : Oui, il décrit afin d'énoncer des règles. Et ces règles sont probablement aussi bien destinées au spectateur, afin qu'il puisse juger des bonnes et des mauvaises tragédies, qu’au fabricant de tragédie.

$F D$ : Mais les spectateurs ne jugent pas selon des normes, ils apprécient dans l'immédiateté $\mathrm{du}$ spectacle, ils aiment ou n'aiment pas.

$A M$ : C'est néanmoins ce que veut faire Aristote, hiérarchiser les bonnes et les mauvaises manières de faire des pièces de théâtre.

$F D:$ Il le fait précisément en dehors de toute réalité.

$A M$ : On pourrait dire que pour Aristote le propos est peut-être en dernière instance anthropologique. Il tente de mesurer ce qui, dans l'évolution donnée d'un art, correspond le mieux à ses yeux à l'épanouissement de la nature humaine.

$F D$ : La question, c'est pourquoi il ne s'est pas contenté de classer les animaux, et pourquoi il lui a fallu venir faire irruption dans des domaines où il n'y connaissait rien, du moins de façon empirique.

$A M$ : Pourtant, si vous le comparez à un Platon, auquel je pense que vous accorderez qu'il connaît bien les rites de sa cité, et en particulier ceux qui peuvent entourer le théâtre, il y a chez Aristote un souci du détail de tel ou tel scénario, de telle ou telle pièce, de telle ou telle pratique scénographique, de telle représentation où une statue est tombée sur un acteur - un souci du concret de cette pratique qui est même étonnant pour un philosophe, ne trouvez-vous pas? 
$F D$ : Tous les lettrés procèdent ainsi et, comme eux, il puise dans des recueils d'anecdotes qui servent aux orateurs. Moi, ce qui m'étonne, c'est qu'il n'y avait aucune raison technique de faire un tel manuel de poétique. Les gens n'ont pas besoin qu'on rende compte d'une pratique qu'ils connaissent très bien, car ils ont vu des dizaines de tragédies et ont participé aux répétitions comme choreutes. Il y a plein d'Athéniens tout à fait capables de faire des tragédies sans avoir besoin de lire Aristote.

$A M$ : Dans ces conditions, on pourrait étendre votre propos à tous les traités d'Aristote. Ainsi l'Éthique à Nicomaque : quelle est cette prétention à croire éduquer des gouvernants, quand les gouvernants n'ont pas attendu Aristote pour gouverner? Pourtant, c'est bien ce qu'Aristote semble avoir l'ambition de faire, et nous savons qu'il a eu l'occasion de le mettre en pratique avec Alexandre.

$M B H$ : Peut-être est-ce également lié au fait qu'il dirige une école et qu'il entend y attirer des élèves? N'y a-t-il pas là une dimension institutionnelle?

$F D$ : Oui, mais aussi qu'il veut être athénien, participer. Personne ne l'aime, on le chasse.

$M B H$ : Depuis longtemps vous vous réclamez d'une méthode anthropologique. Est-ce le cas encore dans ce livre?

$F D$ : Oui, dans la mesure où j'essaye de faire fonctionner la tragédie comme une pratique propre à une culture, saisie dans une anthropologie historique. Ensuite, l'attention au rituel, le fait de ne pas vouloir mettre de côté la dimension religieuse relève aussi de ce type d'approche.

$M B H$ : Lorsque ensuite vous passez à Molière, à Goldoni, est-ce que vous tenez encore à cette étiquette?

$F D$ : Là, non, l'étude est beaucoup plus rapide et superficielle ; il ne peut être question de faire autre chose qu' une vague histoire sociale. Il y a un effort anthropologique en ce qui concerne la Grèce, en ce qui concerne Rome, pour le reste je n’ai pas la formation et la culture nécessaires.

$M B H$ : Qu'en est-il de la réception du livre ? Vous avez voulu vous adresser à un public plus large en choisissant la collection « Libelles ».

$F D$ : Je ne sais pas, on verra; on n'est pas à une époque où ce genre de livre est attendu frénétiquement. Pour ce qui est des aristotéliciens, ici à Paris VII, ils sont plutôt d'accord ; les hellénistes aussi, ce qui m’a plutôt étonnée. Et m’a un peu rassurée. Ce qui est dommage aujourd'hui, c'est que la culture grecque étant moins partagée, le sens critique a diminué et on tend à croire le spécialiste de grec sur parole. La vulgate est de plus en plus opaque. Et des choses restent enfermées dans les débats entre spécialistes, alors que les enjeux sont plus larges que cela. Il faudrait ménager un accès plus populaire à l'Antiquité. Ce qui est un projet aussi pour les metteurs en scène.

Malika BASTIN-HAMmOU \& Arnaud MaCÉ

hammou@univ-tlse2.fr

amace@univ-fcomte.fr 